\title{
ON THE LEFT IDEAL \\ IN THE UNIVERSAL ENVELOPING ALGEBRA \\ OF A LIE GROUP GENERATED \\ BY A COMPLEX LIE SUBALGEBRA
}

\author{
JUAN TIRAO
}

(Communicated by Roe W. Goodman)

\begin{abstract}
Let $G_{0}$ be a connected Lie group with Lie algebra $g_{0}$ and let $h$ be a Lie subalgebra of the complexification $g$ of $g_{0}$. Let $C^{\infty}\left(G_{0}\right)^{h}$ be the annihilator of $h$ in $C^{\infty}\left(G_{0}\right)$ and let $\mathscr{A}=\mathscr{A}\left(C^{\infty}\left(G_{0}\right)^{h}\right)$ be the annihilator of $C^{\infty}\left(G_{0}\right)^{h}$ in the universal enveloping algebra $\mathscr{U}(g)$ of $g$. If $h$ is the complexification of the Lie algebra $h_{0}$ of a Lie subgroup $H_{0}$ of $G_{0}$ then $\mathscr{A}=\mathscr{U}(g) h$ whenever $H_{0}$ is closed, is a known result, and the point of this paper is to prove the converse assertion. The paper has two distinct parts, one for $C^{\infty}$, the other for holomorphic functions. In the first part the Lie algebra $\bar{h}_{0}$ of the closure of $H_{0}$ is characterized as the annihilator in $g_{0}$ of $C^{\infty}\left(G_{0}\right)^{h}$, and it is proved that $h_{0}$ is an ideal in $\bar{h}_{0}$ and that $\bar{h}_{0}=h_{0} \oplus v$ where $v$ is an abelian subalgebra of $\bar{h}_{0}$. In the second part we consider a complexification $G$ of $G_{0}$ and assume that $h$ is the Lie algebra of a closed connected subgroup $H$ of $G$. Then we establish that $\mathscr{A}\left(\mathscr{O}(G)^{h}\right)=\mathscr{U}(g) h$ if and only if $G / H$ has many holomorphic functions. This is the case if $G / H$ is a quasi-affine variety. From this we get that if $H$ is a unipotent subgroup of $G$ or if $G$ and $H$ are reductive groups then $\mathscr{A}\left(C^{\infty}\left(G_{0}\right)^{h}\right)=\mathscr{U}(g) h$.
\end{abstract}

\section{THE SMOOTH SETTING}

Let $G_{0}$ be a (connected) Lie group with Lie algebra $g_{0}$. Let $h$ be a Lie subalgebra of the complexification $g$ of $g_{0}$. Let $\mathscr{U}(g)$ be the universal enveloping algebra of $g$ viewed as the algebra of all left invariant differential operators on $G_{0}$. Hence the space $C^{\infty}\left(G_{0}\right)$ of all complex-valued $C^{\infty}$-functions on $G_{0}$ is a left $\mathscr{U}(g)$-module. If $s$ is any subset of $g$ we set

$$
C^{\infty}\left(G_{0}\right)^{s}=\left\{f \in C^{\infty}\left(G_{0}\right): X f=0 \text { for all } x \in s\right\} .
$$

We shall be concerned with the annihilator $\mathscr{A}=\mathscr{A}\left(C^{\infty}\left(G_{0}\right)^{h}\right)$ in $\mathscr{U}(g)$ of the annihilator $C^{\infty}\left(G_{0}\right)^{h}$ of $h$ in $C^{\infty}\left(G_{0}\right)$. Thus

$$
\mathscr{A}=\left\{D \in \mathscr{U}(g): D f=0 \text { for all } f \in C^{\infty}\left(G_{0}\right)^{h}\right\} .
$$

Received by the editors October 2, 1991 and, in revised form, November 4, 1992.

1991 Mathematics Subject Classification. Primary 22E10, 22E15, 32M10; Secondary 20G20.

Key words and phrases. Lie subgroup, closure, annihilator, complexification, $C^{\infty}$ and holomorphic functions, quasi-affine variety, reductive and unipotent groups. 
Clearly $\mathscr{A}$ is a left ideal of $\mathscr{U}(g)$ containing the left ideal $\mathscr{U}(g) h$ generated by $h$. We are interested in discussing when $\mathscr{A}$ is equal to $\mathscr{U}(g) h$.

Suppose $h$ is the complexification of $h_{0}=h \cap g_{0}, h_{0}$ being the Lie algebra of a closed connected subgroup $H_{0}$ of $G_{0}$. Then it is easy to see that in this case $\mathscr{A}=\mathscr{U}(g) h$. Let us go over the proof of this fact (cf. [5, Lemma 2.5, Chapter X]), because we shall make use of a similar argument.

If $V$ is a finite-dimensional complex vector space, $S(V)$ will denote the symmetric algebra over $V$ and $\lambda$ will denote the symmetrization mapping of $S(g)$ onto $\mathscr{U}(g)$. Let $m_{0} \subset g_{0}$ be a subspace such that $g_{0}=m_{0} \oplus h_{0}$ and let $m \subset g$ be its complexification. Then it is well known (cf. [3, Proposition 2.4 .14$, p. 82]) that $\lambda$ induces a linear isomorphism between $S(m) \otimes S(h)$ and $\mathscr{U}(g)$. Therefore we have

$$
\begin{aligned}
\mathscr{U}(g) & =\lambda(S(m)) \cdot \lambda(S(h)) \\
& =\lambda(S(m)) \cdot(\mathscr{U}(h) h \oplus \mathbb{C} \cdot 1)=\mathscr{U}(g) h \oplus \lambda(S(m)) .
\end{aligned}
$$

Let $X_{1}, \ldots, X_{p}$ be a basis of $g_{0}$ such that $X_{i} \in m_{0}$ for $i=1, \ldots, n$. If $\pi$ denotes the natural mapping of $G_{0}$ onto $G_{0} / H_{0}$, for a suitable open neighbourhood $U$ of 0 in $m_{0}$, the mapping

$$
\pi\left(\exp \left(x_{1} X_{1}+\cdots+x_{n} X_{n}\right)\right) \mapsto\left(x_{1}, \ldots, x_{n}\right)
$$

is a coordinate system on the open neighbourhood $\pi(\exp U)$ of the origin $o$ in $G_{0} / H_{0}$.

If $P \in S(g)$ is identified with a polynomial in the basis elements $X_{1}, \ldots, X_{p}$ and $\partial_{1}, \ldots, \partial_{p}$ denote the partial derivatives with respect to the variables $\left(x_{1}, \ldots, x_{p}\right)$, then (cf. [5, Chapter X, $\left.\left.\S 2,16\right]\right)$

$$
[\lambda(P) f](g)=\left[P\left(\partial_{1}, \ldots, \partial_{p}\right) f\left(g \exp \left(x_{1} X_{1}+\cdots+x_{p} X_{p}\right)\right)\right](0)
$$

for each $g \in G_{0}, f \in C^{\infty}\left(G_{0}\right)$.

In order to prove that $\mathscr{A}\left(C^{\infty}\left(G_{0}\right)^{h}\right)=\mathscr{U}(g) h$ let $P \in S(m), P \neq 0$. There exists $f^{*} \in C^{\infty}(U)$ such that

$$
\left[P\left(\partial_{1}, \ldots, \partial_{n}\right) f^{*}\left(x_{1} X_{1}+\cdots+x_{n} X_{n}\right)\right](0) \neq 0 .
$$

Using a partition of unity argument, it is clear that there exists a function $f \in C^{\infty}\left(G_{0} / H_{0}\right)$ such that $f\left(\pi\left(\exp \left(x_{1} X_{1}+\cdots+x_{n} X_{n}\right)\right)\right)=f^{*}\left(x_{1} X_{1}+\cdots+x_{n} X_{n}\right)$ for all sufficiently small $x_{i}$. From (2) follows

$$
[\lambda(P)(f \circ \pi)](e)=\left[P\left(\partial_{1}, \ldots, \partial_{n}\right) f^{*}\left(x_{1} X_{1}+\cdots+x_{n} X_{n}\right)\right](0) \neq 0,
$$

but since $(f \circ \pi) \in C^{\infty}\left(G_{0}\right)^{h}$ it is clear from (1) that $\mathscr{A}=\mathscr{U}(g) h$.

Thus we have

Theorem 1. Suppose $h$ is the complexification of the Lie algebra of a closed connected Lie subgroup $H_{0}$ of $G_{0}$. Then

$$
\mathscr{A}\left(C^{\infty}\left(G_{0}\right)^{h}\right)=\mathscr{U}(g) h .
$$

Corollary 2. Suppose $h$ is the complexification of the Lie algebra of a connected Lie subgroup $H_{0}$ of $G_{0}$. Let $\bar{h}$ be the complexification of the Lie algebra of the closure $\bar{H}_{0}$ of $H_{0}$. Then

$$
\mathscr{A}\left(C^{\infty}\left(G_{0}\right)^{h}\right)=\mathscr{U}(g) \bar{h} .
$$


Proof. If $K_{0}$ is a subgroup of $G_{0}$ we denote by $C^{\infty}\left(G_{0}\right)^{K_{0}}$ the space of all $C^{\infty}$-functions on $G_{0}$ which are right invariant by all elements in $K_{0}$. It is easy to see that

$$
C^{\infty}\left(G_{0}\right)^{h}=C^{\infty}\left(G_{0}\right)^{H_{0}}=C^{\infty}\left(G_{0}\right)^{\bar{H}_{0}}=C^{\infty}\left(G_{0}\right)^{\bar{h}} .
$$

Thus

$$
\mathscr{A}\left(C^{\infty}\left(G_{0}\right)^{h}\right)=\mathscr{A}\left(C^{\infty}\left(G_{0}\right)^{\bar{h}}\right)=\mathscr{U}(g) \bar{h} .
$$

This completes the proof of the corollary.

If $\mathscr{F}$ is any subset of $C^{\infty}\left(G_{0}\right)$ we define

$$
a_{0}(\mathscr{F})=\left\{X \in g_{0}: X f=0 \text { for all } f \in \mathscr{F}\right\} .
$$

It is easy to verify that $a_{0}(\mathscr{F})$ is a Lie subalgebra of $g_{0}$.

Corollary 3. A Lie subalgebra $h_{0}$ of $g_{0}$ is the Lie algebra of a closed Lie subgroup of $G_{0}$ if and only if

$$
a_{0}\left(C^{\infty}\left(G_{0}\right)^{h_{0}}\right)=h_{0} .
$$

Proof. Let $h$ be the complexification of $h_{0}$. Then

$$
a_{0}\left(C^{\infty}\left(G_{0}\right)^{h_{0}}\right)=\mathscr{A}\left(C^{\infty}\left(G_{0}\right)^{h}\right) \cap g_{0} .
$$

Let $H_{0}$ be the connected Lie subgroup of $G_{0}$ with Lie algebra $h_{0}$ and let $\bar{h}_{0}$ be the Lie algebra of its closure $\bar{H}_{0}$. Then, by Corollary 2 , we have

$$
a_{0}\left(C^{\infty}\left(G_{0}\right)^{h_{0}}\right)=\mathscr{U}(g) \bar{h} \cap g_{0}=\bar{h}_{0} .
$$

Thus $H_{0}$ is closed if and only if $a_{0}\left(C^{\infty}\left(G_{0}\right)^{h_{0}}\right)=h_{0}$. Since the connected component of a closed subgroup is closed, the corollary is proved.

Definition. If $h_{0}$ is a Lie subalgebra of $g_{0}$ we set $\bar{h}_{0}=a_{0}\left(C^{\infty}\left(G_{0}\right)^{h_{0}}\right)$.

Then as it was pointed out above $\bar{h}_{0}$ is the Lie algebra of the closure $\bar{H}_{0}$ of the connected Lie subgroup $H_{0}$ corresponding to $h_{0}$.

Corollary 4. The family of all subalgebras $a_{0}(\mathscr{F})$, where $\mathscr{F}$ is any subset of $C^{\infty}\left(G_{0}\right)$ is precisely the family of all subalgebras of $g_{0}$ which correspond to the closed subgroups of $G_{0}$.

Proof. Given $\mathscr{F} \subset C^{\infty}\left(G_{0}\right)$ let $H_{0}$ be the connected Lie subgroup of $G_{0}$ with Lie algebra $h_{0}=a_{0}(\mathscr{F})$. Since $\mathscr{F} \subset C^{\infty}\left(G_{0}\right)^{h_{0}}$ we have

$$
h_{0} \supseteq a_{0}\left(C^{\infty}\left(G_{0}\right)^{h_{0}}\right) \supseteq h_{0} \text {. }
$$

Thus from Corollary 3 it follows that $H_{0}$ is closed.

Conversely if $h_{0}$ is the Lie algebra of a closed subgroup of $G_{0}$ then, by Corollary $3, h_{0}=a_{0}\left(C^{\infty}\left(G_{0}\right)^{h_{0}}\right)$ which completes the proof of our statement.

In order to emphasize the characterization of the Lie algebra of the closure of a connected Lie subgroup $H_{0}$ of $G_{0}$ as the annihilator in $g_{0}$ of $C^{\infty}\left(G_{0}\right)^{h_{0}}$, we will consider some examples.

Example 1. Let $G_{0}=\mathbb{R}^{2} / \mathbb{Z}^{2}$ be a two-dimensional torus. We identify the Lie algebra of $G_{0}$ with $\mathbb{R}^{2}$ and we denote with $\pi$ the natural mapping of $\mathbb{R}^{2}$ onto $G_{0}$. Given $(a, b) \in \mathbb{R}^{2},(a, b) \neq 0$, let $h_{0}$ be the one-dimensional subalgebra of $\mathbb{R}^{2}$ spanned by $(a, b)$.

If $a / b$ or $b / a$ are rational numbers we shall prove, using the criterion of Corollary 3 , that $h_{0}$ is the Lie algebra of a closed subgroup of $G_{0}$. In fact, we 
may assume that $a, b \in \mathbb{Z}$. There exists $f \in C^{\infty}\left(G_{0}\right)$ such that $(f \circ \pi)(x, y)=$ $e^{2 \pi i(b x-a y)}$ for all $(x, y) \in \mathbb{R}^{2}$. If $X=(c, d) \in \mathbb{R}^{2}$ then

$$
[X f](\pi(x, y))=\left\{\frac{d}{d t} e^{2 \pi i(b(x+c t)-a(y+d t))}\right\}_{t=0}=2 \pi i(b c-\mathrm{ad}) f(\pi(x, y)) .
$$

Thus $X f=0$ if and only if $X \in h_{0}$, in other words $a_{0}(\{f\})=h_{0}$.

On the other hand if $a / b$ is irrational we shall prove that $C^{\infty}\left(G_{0}\right)^{h_{0}}=\mathbb{C}$ which implies that the connected Lie subgroup of $G_{0}$ corresponding to $h_{0}$ is dense (cf. (3)). Let $X=(a, b)$ and let $f \in C^{\infty}\left(G_{0}\right)$ such that $X f=0$. Then $F=f \circ \pi$ satisfies $a \frac{\partial F}{\partial x}+b \frac{\partial F}{\partial y}=0$. If we introduce the new linear coordinates $(u, v)$ on $\mathbb{R}^{2}$, defined by $x=b u+a v, y=-a u+b v$, then $\frac{\partial F}{\partial v}=0$, hence $F(u, v)=\varphi(u), \varphi \in C^{\infty}(\mathbb{R})$. Therefore $F(x, y)=\varphi\left((b x-a y) /\left(a^{2}+b^{2}\right)\right)$ for all $(x, y) \in \mathbb{R}^{2}$. But $\varphi$ is constant on the subgroup $\left\{(b m+a n) /\left(a^{2}+b^{2}\right)\right.$ : $m, n \in \mathbb{Z}\}$ of $\mathbb{R}$. This subgroup is either closed (and isomorphic to $\mathbb{Z}$ ) or it is dense in $\mathbb{R}$. In the first case the ratio of two of its nonzero elements would be rational, which is impossible since $a / b$ is irrational. Therefore $\varphi$ is constant and a fortiori $f$ is constant.

Example 2 (cf. [2, p. 127]). Let $G_{0}$ be a connected, simply connected Lie group with Lie algebra $g_{0}$. If $h_{0}$ is an ideal in $g_{0}$, then the connected Lie subgroup $H_{0}$ of $G_{0}$ with Lie algebra $h_{0}$ is closed in $G_{0}$.

In fact let $K_{0}$ be a connected Lie group with Lie algebra $g_{0} / h_{0}$. Let $\sigma: G_{0} \rightarrow$ $K_{0}$ be the analytic homomorphism whose differential $d \sigma: g_{0} \rightarrow g_{0} / h_{0}$ is the natural mapping. Let $\mathscr{F}=\left\{f \circ \sigma: f \in C^{\infty}\left(K_{0}\right)\right\}$. Then

$$
\begin{aligned}
X \in a_{0}(\mathscr{F}) & \Leftrightarrow 0=X(f \circ \sigma)=d \sigma(X) f \quad \text { for all } f \in C^{\infty}\left(K_{0}\right) \\
& \Leftrightarrow d \sigma(X)=0 \Leftrightarrow X \in h_{0} .
\end{aligned}
$$

Thus from Corollary 4 it follows that $H_{0}$ is closed in $G_{0}$.

Example 3. Let $G_{0}$ be a connected Lie group with Lie algebra $g_{0}$ and let $\tau: G_{0} \rightarrow \mathrm{GL}(V)$ be a finite-dimensional real or complex representation of $G_{0}$. If $h_{0}$ is a subalgebra of $g_{0}$ and $W$ is an $h_{0}$-invariant linear subspace of $V$ then $W$ is also $\bar{h}_{0}$ - invariant. In particular $h_{0}$ is an ideal of $\bar{h}_{0}$.

To prove the above statement let us consider the Grassmannian $M$ of all linear subspaces of $V$ of the same dimension as $W$ with the action of $G_{0}$ on $M$ defined by $g \cdot A=\tau(g)(A), g \in G_{0}, A \in M$.

Given any $\varphi \in C^{\infty}(M)$ let $f \in C^{\infty}\left(G_{0}\right)$ be defined by $f(g)=\varphi(g \cdot W)$, $g \in G_{0}$. If $X \in g_{0}$ then

$$
[X f](g)=\left\{\frac{d}{d t} \varphi(g \exp t X \cdot W)\right\}_{t=0} .
$$

Thus if $X \in h_{0}$ then $X f=0$. Therefore $X f=0$ for all $X \in \bar{h}_{0}$. Hence $\varphi(\exp t X \cdot W)=\varphi(W)$ for all $t \in \mathbb{R}, X \in \bar{h}_{0}, \varphi \in C^{\infty}(M)$, which implies that $W$ is $\bar{h}_{0}$-invariant.

Finally if we consider the adjoint representation of $G_{0}$ then $h_{0}$ is an $\operatorname{ad}\left(h_{0}\right)$ invariant subspace of $g_{0}$ and therefore it is also $\operatorname{ad}\left(\bar{h}_{0}\right)$-invariant, which is equivalent to say that $h_{0}$ is an ideal of $\bar{h}_{0}$.

Example 4 (cf. [10, p. 615]). Let $\tau: G_{0} \rightarrow \mathrm{GL}(V)$ be a finite-dimensional complex representation of $G_{0}$ such that $d \tau$ is a faithful representation of $g_{0}$. If 
$h_{0}$ is a semisimple Lie subalgebra of $g_{0}$ then the corresponding connected Lie subgroup $H_{0}$ of $G_{0}$ is closed.

From the previous example, we know that $h_{0}$ is an ideal in $\bar{h}_{0}$, thus any element in $\bar{h}_{0}$ induces a derivation of $h_{0}$. Since the only derivations of a semisimple Lie algebra are the inner ones we see that $\bar{h}_{0}=h_{0}+s_{0}$, where $s_{0}$ is the centralizer of $h_{0}$ in $\bar{h}_{0}$. Moreover the sum is direct because $h_{0}$ is centerless.

Let $W$ be an $h_{0}$-irreducible complex subspace of $V$. Then $W$ is also $\bar{h}_{0^{-}}$ invariant (cf. Example 3). From Schur's lemma we know that $\left.d \tau(X)\right|_{W}$ is a scalar for all $X \in s_{0}$. Now take a projection $P$ of $V$ onto $W$ commuting with $d \tau\left(h_{0}\right)$ and let $\tilde{\tau}: G_{0} \rightarrow \operatorname{End}(W)$ be defined by $P \tau(g) P=0 \oplus \tilde{\tau}(g), g \in G_{0}$, according to the decomposition $V=\operatorname{ker} P \oplus W$. If $f=\operatorname{det} \tilde{\tau}$ and $X \in \bar{h}_{0}$ we have

$$
[X f](g)=\left\{\frac{d}{d t} \operatorname{det}(\tilde{\tau}(g) \tilde{\tau}(\exp t X))\right\}_{t=0}=f(g) \operatorname{tr}\left(\left.d \tau(X)\right|_{W}\right)
$$

Since $h_{0}$ coincides with its derived algebra it follows that $f \in C^{\infty}\left(G_{0}\right)^{h_{0}}$ and therefore $X f=0$ for all $X \in \bar{h}_{0}$, which implies that $\operatorname{tr}\left(\left.d \tau(X)\right|_{W}\right)=0$ for all $X \in \bar{h}_{0}$. Thus $\left.d \tau(X)\right|_{W}=0$ if $X \in s_{0}$. Since any finite-dimensional representation of a semisimple Lie algebra is completely reducible, we obtain that $d \tau(X)=0$, if $X \in s_{0}$, which in turn implies that $X=0$. Therefore $\bar{h}_{0}=h_{0}$ which is equivalent to $H_{0}$ being closed (Corollary 3 ).

As a corollary of this example we get the following results:

(i) If $G_{0}$ is a connected Lie group which is either simply connected or compact, and $h_{0}$ is a semisimple Lie subalgebra of $g_{0}$ then the corresponding connected Lie subgroup $H_{0}$ of $G_{0}$ is closed (Mostow [10, p. 615]).

(ii) If $G_{0}$ is a group of matrices and $h_{0}$ is a semisimple Lie subalgebra of $g_{0}$ then $H_{0}$ is closed (see also [4]).

Theorem 5. Let $G_{0}$ be a connected Lie group and let $h_{0}$ be a Lie subalgebra of the Lie algebra $g_{0}$ of $G_{0}$. Then

$$
\bar{h}_{0}=\sum_{Y \in h_{0}} a_{0}\left(C^{\infty}\left(G_{0}\right)^{Y}\right)
$$

where $C^{\infty}\left(G_{0}\right)^{Y}=\left\{f \in C^{\infty}\left(G_{0}\right): Y f=0\right\}$. Moreover there exists an abelian subalgebra $v$ such that $\bar{h}_{0}=h_{0} \oplus v$.

Proof. We shall make use of the following result of Gotô [4, Theorem 1, p. 114]. Let $H_{0}$ be the connected Lie subgroup of $G_{0}$ with Lie algebra $h_{0}$, let $K$ be a maximal compact subgroup of $\bar{H}_{0}$, and let $U$ be the radial of $K$. Then $\bar{H}_{0}=U H_{0}$ and if we put $U_{1}=U \cap H_{0}$ we have $\bar{U}_{1}=U$.

Let $u$ and $u_{1}$ be, respectively, the Lie algebras of $U$ and $U_{1}$. Then

$$
\sum_{Y \in h_{0}} a_{0}\left(C^{\infty}\left(G_{0}\right)^{Y}\right) \supset h_{0}+\sum_{Y \in u_{1}} a_{0}\left(C^{\infty}\left(G_{0}\right)^{Y}\right) .
$$

But since $U$ is a torus and $\bar{U}_{1}=U$ the well-known theorem of Kronecker implies that there exists $Y \in u_{1}$ such that $a_{0}\left(C^{\infty}\left(G_{0}\right)^{Y}\right)=u$. Hence

$$
\sum_{Y \in h_{0}} a_{0}\left(C^{\infty}\left(G_{0}\right)^{Y}\right) \supset h_{0}+u=\bar{h}_{0} \text {. }
$$

Since the other inclusion is clear the first assertion is proved. 
Finally by taking any direct complement $v$ of $u_{1}$ in $u$ the last statement follows.

Corollary 6 (Gotô [4]). A necessary and sufficient condition for a connected Lie subgroup $H_{0}$ of $G_{0}$ to be closed is that $H_{0}$ contains the closure of every oneparameter subgroup of $H_{0}$.

\section{THE HOLOMORPHIC SETTING}

Since the problem under consideration involves a complex Lie subalgebra of the complexification $g$ of the Lie algebra of $G_{0}$, it is reasonable to think of a complexification $G$ of $G_{0}$. But generally a Lie group has no complexification, unless it has a faithful finite-dimensional representation. A weaker demand is to look for an analytic homomorphism $\phi: G_{0} \rightarrow G$ into a (connected) complex Lie group $G$ such that $d \phi$ is injective. For $G_{0}$ a Lie group with a finite number of connected components, Hochschild and Mostow [7] have constructed a complex Lie group $G$ and an analytic homomorphism $\phi: G_{0} \rightarrow G$ such that for any finite-dimensional representation $D$ of $G_{0}$, there exists a unique holomorphic representation $\widetilde{D}$ of $G$ satisfying $D=\widetilde{D} \circ \phi$. This is called the universal complexification of $G_{0}$. Moreover it is known (cf. [11, Corollary to Proposition 10]) that if $G_{0}$ has a finite-dimensional representation $\tau$ such that $d \tau$ is faithful (for example if $G_{0}$ is semisimple), then the universal complexification $\phi: G_{0} \rightarrow G$ of $G_{0}$ is such that $d \phi$ is injective and the Lie algebra $g$ of $G$ coincides with the complexification of $d \phi\left(g_{0}\right)$. From now on we shall assume that $\phi: G_{0} \rightarrow G$ satisfies these two conditions and we shall identify $g_{0}$ with $d \phi\left(g_{0}\right)$. Then for all $f \in \mathscr{O}(G)$ and $D \in \mathscr{U}(g)$ we have

$$
D(f \circ \phi)=(D f) \circ \phi \text {. }
$$

We must be careful; this does not hold for all $f \in C^{\infty}(G)$. For example if $\phi: \mathbb{R} \rightarrow \mathbb{C}$ is the canonical injection and $t$ and $z=x+i y$ are the usual coordinate functions on $\mathbb{R}$ and $\mathbb{C}$, respectively, then $d t$ is identified with $\frac{\partial}{\partial x}$ and $i \frac{d}{d t}$ with $\frac{\partial}{\partial y}$. Now if we take $f(x, y)=y$ then $f \circ \phi \equiv 0$, thus $\frac{\partial}{\partial y}(f \circ \phi) \equiv 0$ while $\left(\frac{\partial}{\partial y} f\right) \circ \phi \equiv 1$.

In particular if $f \in \mathscr{O}(G)^{h}=\mathscr{O}(G) \cap C^{\infty}(G)^{h}$ then $f \circ \phi \in C^{\infty}\left(G_{0}\right)^{h}$. Also if $D \in \mathscr{A}\left(C^{\infty}\left(G_{0}\right)^{h}\right)$ then

$$
[D f](e)=[D(f \circ \phi)](e)=0
$$

for all $f \in \mathscr{O}(G)^{h}$. But $\mathscr{O}(G)^{h}$ is stable under left multiplication by any $x \in G$, thus

$$
[D f](x)=[D(f \circ L(x))](e)=0
$$

for all $f \in \mathscr{O}(G)^{h}$. Therefore we have

$$
\mathscr{U}(g) h \subset \mathscr{A}\left(C^{\infty}\left(G_{0}\right)^{h}\right) \subset \mathscr{A}\left(\mathscr{O}(G)^{h}\right),
$$

where $\mathscr{A}\left(\mathscr{O}(G)^{h}\right)=\left\{D \in \mathscr{U}(g): D f=0\right.$ for all $\left.f \in \mathscr{O}(G)^{h}\right\}$.

Suppose $H$ is a closed complex subgroup of $G$. We say that the homogeneous space $X=G / H$ has many holomorphic functions if there are holomorphic independent functions $f_{1}, \ldots, f_{n} \in \mathscr{O}(X)$ (i.e., $d f_{1} \wedge \cdots \wedge d f_{n} \neq 0$ ), where $n=\operatorname{dim}_{\mathbb{C}} X$. 
Theorem 7. Suppose $h$ is the Lie algebra of a closed connected complex subgroup $H$ of $G$. Then

$$
\mathscr{A}\left(\mathscr{O}(G)^{h}\right)=\mathscr{U}(g) h
$$

if and only if $G / H$ has many holomorphic functions.

Proof. Let $m \subset g$ be a complex subspace such that $g=m \oplus h$, and let $X_{1}, \ldots, X_{p}$ be a basis of $g$ such that $X_{i} \in m$ for $i=1, \ldots, n$. If $\pi$ denotes the natural mapping of $G$ onto $G / H$, for a suitable open neighbourhood $U$ of 0 in $m$ the mapping

$$
\pi\left(\exp \left(x_{1} X_{1}+\cdots+x_{n} X_{n}\right)\right) \mapsto\left(x_{1}, \ldots, x_{n}\right)
$$

is a holomorphic coordinate system on the open neighbourhood $\pi(\exp U)$ of the origin $O$ in $G / H$.

If $P \in S(g)$ is identified with a polynomial in the basis elements $X_{1}, \ldots, X_{p}$ and $\partial_{1}, \ldots, \partial_{p}$ denote the partial derivatives with respect to the complex variables $\left(x_{1}, \ldots, x_{p}\right)$ then (cf. (2))

$$
[\lambda(P) f](g)=\left[P\left(\partial_{1}, \ldots, \partial_{p}\right) f\left(g \exp \left(x_{1} X_{1}+\cdots+x_{p} X_{p}\right)\right)\right](0)
$$

for each $g \in G, f \in \mathscr{O}(G)$.

Let us assume now that $G / H$ has many holomorphic functions. Then there exist $y_{1}, \ldots, y_{n} \in \mathscr{O}(G / H)$ such that the map $x \mapsto\left(y_{1}(x), \ldots, y_{n}(x)\right)$ defines a holomorphic coordinate system in a neighbourhood $V$ of the origin and such that $y_{j}(o)=0$ for $j=1, \ldots, n$. Thus given $P \in S(m), P \neq 0$, there exists $P^{\prime} \in S(m), P^{\prime} \neq 0$ such that

$$
\begin{array}{r}
{\left[P\left(\partial_{1}, \ldots, \partial_{n}\right) f\left(\pi\left(\exp \left(x_{1} X_{1}+\cdots+x_{n} X_{n}\right)\right)\right)\right](0)} \\
\quad=\left[P^{\prime}\left(\frac{\partial}{\partial y_{1}}, \ldots, \frac{\partial}{\partial y_{n}}\right) f^{*}\left(y_{1}, \ldots, y_{n}\right)\right](0)
\end{array}
$$

for all $f \in \mathscr{O}(G / H)$, where $f^{*}\left(y_{1}(x), \ldots, y_{n}(x)\right)=f(x)$ for all $x \in V$. Now it is clear that we may choose $f \in \mathscr{O}(G / H)$ such that

$$
[\lambda(P)(f \circ \pi)](e) \neq 0 .
$$

Since $\mathscr{U}(g)=\mathscr{U}(g) h \oplus \lambda(S(m))$ (see (1)) and $f \circ \pi \in \mathscr{O}(G)^{h}$ it follows that $\mathscr{A}\left(\mathscr{O}(G)^{h}\right)=\mathscr{U}(g) h$.

Conversely, let us assume that $\mathscr{A}\left(\mathscr{O}(G)^{h}\right)=\mathscr{U}(g) h$. Then making the natural identification between $m$ and the holomorphic tangent space of $G / H$ at $o$ we know that

$$
\left\{X \in m: d f_{o}(X)=0 \text { for all } f \in \mathscr{O}(G / H)\right\}=\{0\} .
$$

This is equivalent to say that the linear map $f \mapsto d f_{o}$ from $\mathscr{O}(G / H)$ into $m^{*}$ is surjective. Thus if $X_{1}, \ldots, X_{n}$ is a basis of $m$ there exist $f_{1}, \ldots, f_{n} \in$ $\mathscr{O}(G / H)$ such that $d\left(f_{i}\right)_{o}\left(X_{j}\right)=\delta_{i j}, i, j=1, \ldots, n$. This completes the proof that $G / H$ has many holomorphic functions.

In those cases where $\mathscr{U}(g) h=A\left(\mathscr{O}(g)^{h}\right)$ we certainly have (see (4)) $\mathscr{U}(g) h=$ $A\left(C^{\infty}\left(G_{0}\right)^{h}\right)$, but the converse need not be true. To see this let us consider the following example. 
Example 5. Let $G_{0}$ be a connected noncompact semisimple Lie group and let $K_{0}$ be a maximal compact subgroup of $G_{0}$. Assume that $G_{0} / K_{0}$ is an Hermitian symmetric space. If $g_{0}=k_{0} \oplus p_{0}$ is the Cartan decomposition of $g_{0}$ corresponding to $K_{0}$ and $g=k \oplus p$ is the corresponding complexification then $p$ can be identified with the complex tangent space of $G_{0} / K_{0}$ at the origin $o$. Let $p_{+}$and $p_{-}$be the linear subspaces of $p$ which correspond, respectively, to the holomorphic and antiholomorphic tangent spaces of $G_{0} / K_{0}$ at $o$. Thus it is known (cf. [5, Chapter VIII, Section 7]) that $p_{+}$and $p_{-}$are abelian subalgebras of $g$. Also if $G$ denotes the simply connected Lie group with Lie algebra $g$ and $K, P_{-}$are the analytic subgroups of $G$ corresponding to the subalgebras $k$ and $p_{-}$, respectively, then $H=K P_{-}$is a closed subgroup of $G$ and $G_{0} / K_{0}$ can be holomorphically embedded as an open subset of $G / H$. Moreover, $G / H$ is compact.

Now $C^{\infty}\left(G_{0}\right)^{h}=\left\{f \circ \pi: f \in \mathscr{O}\left(G_{0} / K_{0}\right)\right\}$. If $D \in \mathscr{A}\left(C^{\infty}\left(G_{0}\right)^{h}\right)$ then according to (1) $D=D_{1}+D_{2}$ where $D_{1} \in \mathscr{U}(g) h$ and $D_{2} \in \mathscr{U}\left(p_{+}\right)$. But $D_{2}(f \circ \pi)=0$ for all $f \in \mathcal{O}\left(G_{0} / K_{0}\right)$ clearly implies $D_{2}=0$ since $G_{0} / K_{0}$ has many holomorphic functions. Thus

$$
\mathscr{A}\left(C^{\infty}\left(G_{0}\right)^{h}\right)=U(g) h .
$$

On the other hand $\mathscr{O}(G)^{h}=\mathbb{C}$ since $G / H$ is compact and connected. Therefore we have $\mathscr{A}\left(\mathscr{O}(G)^{h}\right)=\mathscr{U}(g) g$.

The complex manifold $X=G / H$ is said to be quasi-affine if it can be equivariantly embedded as a Zariski open set in an affine variety $Y$. If $G$ is a connected complex reductive Lie group and $G / H$ has many holomorphic functions then it can be proved that $X$ carries a quasi-affine structure (see [9, p. 160]). Thus we have

Corollary 8. Suppose $G$ is a connected complex reductive Lie group and $H$ a closed connected complex Lie subgroup with Lie algebra $h$. Then $\mathscr{A}\left(\mathscr{O}(G)^{h}\right)=$ $\mathscr{U}(g) h$ if and only if $G / H$ is a quasi-affine variety.

Results on when $G / H$ carries a quasi-affine structure can be found in $[1,6]$. If $G$ is a connected complex reductive linear Lie group then it has one and only one structure of an affine algebraic group that is compatible with its structure as a complex Lie group. Moreover if $H$ is a connected complex reductive Lie subgroup of $G$ ( $H$ is necessarily closed in $G)$ then $G / H$ can be equipped with affine structures compatible with its structure of complex manifold (see [6, Theorem 3.2]). Therefore we can state

Corollary 9. Let $G$ be a connected complex reductive linear Lie group and let $H$ be a connected complex reductive Lie subgroup with Lie algebra $h$. Then $\mathscr{A}\left(\mathscr{O}(G)^{h}\right)=\mathscr{U}(g) h$.

If $G$ is an (affine) algebraic group over $\mathbb{C}$ and $H$ is a unipotent algebraic subgroup of $G$ then, by a theorem of Chevalley (see [8, Theorem 11.2]), there is a rational representation $\varphi: G \rightarrow \mathrm{GL}(V)$ and a one-dimensional subspace $L$ of $V$ such that

$$
H=\{x \in G: \varphi(x) L=L\} .
$$

Since a unipotent algebraic group has no nontrival one-dimensional representation, if we pick $v \in L, v \neq 0$, we have

$$
H=\{x \in G: \varphi(x) v=v\} \text {. }
$$


In this way we see that the homogeneous space $G / H$ can be identified with the orbit in $V$ of the point $v$. Such an orbit is a quasi-affine variety (being open in its closure). Thus we have

Corollary 10. If $G$ is a linear algebraic group over $\mathbb{C}$ and $H$ is a connected unipotent algebraic subgroup with Lie algebra $h$, then $\mathscr{A}\left(\mathscr{O}(G)^{h}\right)=\mathscr{U}(g) h$.

If a connected complex reductive Lie group $G$ acts holomorphically on $X$, then a theorem of Harish-Chandra (cf. [12, Theorems 4.4.2.1 and 4.4.3.1]) guarantees that the subspace of all holomorphic $G$-finite functions on $X$ is a dense subset of the Fréchet space $\mathscr{O}(X)$. In fact, since $G$ is the complexification of its maximal compact subgroup $K$ (uniquely determined up to conjugacy) the subspace of all holomorphic $G$-finite functions on $X$ coincides with the subspace of all holomorphic $K$-finite functions on $X$. Also if $\widehat{G}$ denotes the set of all equivalence classes of holomorphic irreducible finite-dimensional representations of $G$ and if $V_{\gamma}$ denotes a fixed $G$-module in the class $\gamma \in \widehat{G}$, then it is known that the subspace of all holomorphic $G$-finite functions (under the left regular representation) on $G$ can be identified with $\bigoplus_{\gamma \in \widehat{G}} V_{\gamma} \otimes V_{\gamma^{*}}$. If $h$ is a Lie subalgebra of the Lie algebra of $G, V_{\gamma}^{h}$ will denote the subspace of all $h$-invariants in $V_{\gamma}$.

Theorem 11. Let $G$ be a connected complex reductive Lie group and let $H$ be a connected closed complex Lie subgroup of $G$ with Lie algebra $h$. Then

$$
\mathscr{U}(g) h=\left\{D \in \mathscr{U}(g): D\left(V_{\gamma}^{h}\right)=0 \text { for all } \gamma \in \widehat{G}\right\}
$$

if and only if $G / H$ is a quasi-affine variety.

Proof. The linear map $f \mapsto f \circ \pi$ is a continuous $G$-morphism from $\mathscr{O}(G / H)$ onto $\mathscr{O}(G)^{h}$. Therefore $\bigoplus_{\gamma \in \widehat{G}} V_{\gamma} \otimes V_{\gamma^{*}}^{h}$ is dense in $\mathscr{O}(G)^{h}$. Since any $D \in \mathscr{U}(g)$ defines a continuous endomorphism of $\mathscr{O}(G)$ it follows that

$$
\left\{D \in \mathscr{U}(g): D\left(V_{\gamma}^{h}\right)=0 \text { for all } \gamma \in \widehat{G}\right\}=\mathscr{A}\left(\mathscr{O}(G)^{h}\right) .
$$

Now the theorem follows from Corollary 8 .

Let $g$ be a finite-dimensional complex semisimple Lie algebra and let $g=$ $u \oplus h \oplus u_{-}$be a triangular decomposition of $g$. Let $\hat{g}$ denote the space of all finite-dimensional irreducible representations of $g$. If $\gamma \in \hat{g}$ let $V_{\gamma}$ denote a fixed $g$-module in the class $\gamma$ and let $V_{\gamma}^{u}$ denote the subspace of $V_{\gamma}$ spanned by a highest weight vector in $V_{\gamma}$. A direct consequence of the above theorem is the following undoubtedly known result.

Corollary 12. Let $g$ be a finite-dimensional complex semisimple Lie algebra and let $g=u \oplus h \oplus u_{-}$be a triangular decomposition of $g$. Then

$$
\mathscr{U}(g) u=\left\{D \in \mathscr{U}(g): D\left(V_{\gamma}^{u}\right)=0 \text { for all } \gamma \in \hat{g}\right\} .
$$

Proof. Let $G$ be a simply connected Lie group with Lie algebra $g$ and let $U$ be the connected Lie subgroup of $G$ with Lie algebra $u$. Then $G$ is a linear algebraic group and $U$ is a maximal unipotent subgroup of $G$. Now the corollary follows from Theorem 11 since $G / U$ is a quasi-affine variety. 


\section{ACKNOWLEDGMENT}

The author would like to thank Professor Abdus Salam, the International Atomic Energy Agency, and UNESCO for hospitality at the International Centre for Theoretical Physics, Trieste.

\section{REFERENCES}

1. A. Bialynicki-Birula, G. Hochschild, and G. D. Mostow, Extensions of representations of algebraic linear groups, Amer. J. Math. 85 (1963), 131-144.

2. C. Chevalley, Theory of Lie groups, Vol. 1, Princeton Univ. Press, Princeton, NJ, 1946.

3. J. Dixmier, Algèbres enveloppantes, Gauthier-Villars, Paris, 1974.

4. M. Gotô, Faithful representations of Lie groups. I, Math. Japon. 1 (1948), 107-119.

5. S. Helgason, Differential geometry and symmetric spaces, Academic Press, New York and London, 1962.

6. G. Hochschild and G. D. Mostow, Affine embeddings of complex analytic homogeneous spaces, Amer. J. Math. 87 (1965), 807-839.

7. (1957), 495-542.

8. J. Humphreys, Linear algebraic groups, Springer-Verlag, Berlin, New York, and Heidelberg, 1975.

9. A. T. Huckleberry and E. Oeljeklaus, Homogeneous spaces from a complex analytic viewpoint, Manifolds and Lie Groups, Progr. Math., vol. 14, Birkhäuser, Basel and Boston, MA, 1981, pp. 159-186.

10. G. D. Mostow, The extensibility of local Lie groups of transformations and groups on surfaces, Ann. of Math. (2) 52 (1950), 606-636.

11. M. Sugiura, The Tannaka duality theorem for semisimple Lie groups and the unitarian trick, Manifolds and Lie Groups (Notre Dame, Indiana, 1980), Progr. Math., vol. 14, Birkhäuser, Basel and Boston, MA, 1981, pp. 405-428.

12. G. Warner, Harmonic analysis on semi-simple Lie groups. I, Springer-Verlag, Berlin and New York, 1972.

International Centre for Theoretical Physics, Trieste, Italy

Current address: FAMAF, Universidad Nacional de Córdoba, Ciudad Universitaria, 5000 Córdoba, Argentina

E-mail address: tirao\%maf cor@uunet.uu.net 